\title{
Effect of a 16-week Bikram yoga program on heart rate variability and associated cardiovascular disease risk factors in stressed and sedentary adults: A randomized controlled trial
}

\author{
Zoe L. Hewett ${ }^{1 *}$, Kate L. Pumpa ${ }^{3}$, Caroline A. Smith², Paul P. Fahey ${ }^{1}$ and Birinder S. Cheema ${ }^{1,2}$
}

\begin{abstract}
Background: Chronic activation of the stress-response can contribute to cardiovascular disease risk, particularly in sedentary individuals. This study investigated the effect of a Bikram yoga intervention on the high frequency power component of heart rate variability (HRV) and associated cardiovascular disease (CVD) risk factors (i.e. additional domains of HRV, hemodynamic, hematologic, anthropometric and body composition outcome measures) in stressed and sedentary adults.

Methods: Eligible adults were randomized to an experimental group $(n=29)$ or a no treatment control group $(n=34)$. Experimental group participants were instructed to attend three to five supervised Bikram yoga classes per week for 16 weeks at local studios. Outcome measures were assessed at baseline (week 0 ) and completion (week 17).

Results: Sixty-three adults (37.2 \pm 10.8 years, $79 \%$ women) were included in the intention-to-treat analysis. The experimental group attended $27 \pm 18$ classes. Analyses of covariance revealed no significant change in the high-frequency component of HRV $\left(p=0.912\right.$, partial $\left.\eta^{2}=0.000\right)$ or in any secondary outcome measure between groups over time. However, regression analyses revealed that higher attendance in the experimental group was associated with significant reductions in diastolic blood pressure $\left(p=0.039\right.$; partial $\left.\eta^{2}=0.154\right)$, body fat percentage $\left(p=0.001\right.$, partial $\left.\eta^{2}=0.379\right)$, fat mass $\left(p=0.003\right.$, partial $\left.\eta^{2}=0.294\right)$ and body mass index $\left(p=0.05\right.$, partial $\left.\eta^{2}=0.139\right)$.

Conclusions: A 16-week Bikram yoga program did not increase the high frequency power component of HRV or any other CVD risk factors investigated. As revealed by post hoc analyses, low adherence likely contributed to the null effects. Future studies are required to address barriers to adherence to better elucidate the doseresponse effects of Bikram yoga practice as a medium to lower stress-related CVD risk.
\end{abstract}

Trial registration: Retrospectively registered with Australia New Zealand Clinical Trials Registry ACTRN12616000867493. Registered 04 July 2016.

Keywords: Hatha yoga, obesity, overweight, inactivity, health, metabolic disease, physiological stress

\footnotetext{
* Correspondence: zoehewett@hotmail.com

'School of Science and Health, Western Sydney University, Locked Bag 1797,

Penrith, NSW 2751, Australia

Full list of author information is available at the end of the article
} 


\section{Background}

Chronic psychological stress is associated with increased risk of cardiovascular disease (CVD) and associated mortality [1-3]. During stress, the sympathetic nervous system (SNS) mediates neuroendocrine changes via the hypothalamic-pituitary-adrenal (HPA) axis [4, 5]. This 'fight-or-flight' response includes the release of stress hormones (e.g. cortisol, aldosterone, epinephrine), which in turn increase heart rate, blood pressure, and blood lipid and glucose concentrations, preparing the body for physical exertion. Chronic SNS activity, via this mechanism, can contribute to atherosclerosis and CVD, particularly in individuals who are physically inactive (sedentary) [6, 7].

Heart rate variability (HRV) is the instantaneous variation in heart rhythm due to autonomic nervous system (ANS) influences on the sinoatrial node. Low HRV is associated with a reduced capacity to adjust to environmental demands [8,9] and increased CVD and mortality $[1,9,10]$. Moreover, low HRV is consistently noted in individuals who are sedentary [11], overweight-obese $[12,13]$ and psychologically stressed [3]. Specific components of HRV can denote the relative input of each branch of the ANS. Vagal activity is reflected in the high frequency (HF) spectral power component of HRV [14].

Hatha yoga is a branch of yoga originating from India that emphasizes the performance of physical postures (asanas) [15]. Several studies have reported that hatha yoga can reduce perceived stress [16] and salivary cortisol, a main effector of the SNS and HPA axis pathways [17-19], as well as improve cardiometabolic health [20]. Studies have also reported that a single session of hatha yoga can acutely increase the HF power component of HRV [21-24]. However, the chronic effects of hatha yoga training on HRV remain inconclusive due to a lack of robust clinical trials [25].

Bikram yoga is a specific system of hatha yoga that incorporates a 90-min, unchanging sequence of asanas performed in a heated environment $\left(40.6{ }^{\circ} \mathrm{C}, 40 \%\right.$ humidity) [26]. A randomized controlled trial (RCT) of Bikram yoga reported reduced reactivity to stress (cortisol) in women at risk for obesity-related illnesses [27], while uncontrolled trials have shown that 8-weeks of Bikram yoga can reduce perceived stress in apparently healthy adults [28], reduce blood lipids (i.e. total and low-density lipoprotein cholesterol) and arterial stiffness in healthy adults [29] and improve glucose tolerance, body mass and body mass index (BMI) in obese adults [30]. The heated environment is a cornerstone feature of Bikram yoga and may aid in its effectiveness in abating CVD risk factors. For example, recent prospective data indicates that more frequent sauna bathing is associated with reduced risk of sudden cardiac death, coronary heart disease, and all-cause mortality in males [31]. Further, preliminary evidence suggests that thermal exposure (sauna bathing and spa treatment) may lead to increased resting HRV in healthy subjects and athletes [32, 33].

To date, no study has investigated the effect of Bikram yoga on any HRV outcomes [34]. Therefore, the purpose of this study was to investigate the effect of a 16-week Bikram yoga intervention on the HF power component of HRV and associated CVD risk factors in a population of stressed and sedentary adults. We hypothesized that participants randomized to the intervention would significantly increase the HF power component and experience significant adaptation of associated CVD risk factors, including additional HRV measures (time and frequency domains) and a range of hemodynamic, hematologic, anthropometric and body composition outcomes compared to a no treatment control.

\section{Methods \\ Study design}

This 16-week, parallel-arm RCT compared the outcomes of participants randomized to an experimental group (Bikram yoga) or a no-treatment control group. Primary and secondary outcomes were collected prior to and following the intervention period at weeks 0 and 17, respectively. The Western Sydney University (H10549) and University of Canberra Human Research Ethics Committees (H10549-14/009174) approved all procedures and written informed consent was obtained from all participants.

\section{Participants and recruitment}

Eligibility criteria: (1) Adult (>18 years); (2) sedentary (i.e. $<150 \mathrm{~min}$ of moderate-intensity exercise per week [35] for greater than 6 months); (3) a score > 14 on the stress component of the Depression, Anxiety and Stress Scale (DASS-21) [36] indicating mild stress; (4) no diagnosed chronic diseases; (5) no acute or chronic medical conditions which would make Bikram yoga potentially hazardous (i.e. pregnancy) or primary outcome difficult to assess (i.e. pacemaker influences on HRV); (6) able to attend three to five Bikram yoga classes per week for 16 weeks; (7) cognition and English language sufficient to understand research procedures and provide informed consent; (8) no participation in Bikram yoga in the past 6 months. Original inclusion criteria included waist circumference $\geq 94 \mathrm{~cm}$ for men and $\geq 80 \mathrm{~cm}$ for women but was removed due to low rate of recruitment.

Participants were recruited between August 2014 and September 2015 in the Australian Capital Territory (ACT) using flyers (posted at local community dwellings, and shared via social media) and word of mouth referral. Individuals who contacted the principal investigator and expressed interest were assessed for eligibility 
using a standardized screening process and questionnaires administered via email and telephone interview. An individual who responded 'yes' to any question on the Physical Activity Readiness Questionnaire (PAR-Q) [37] required medical clearance prior to participating in the trial. Data collection was completed in January 2016.

\section{Sample size}

To date there has been no study investigating the effect of Bikram yoga on parameters of HRV. Therefore, we based our sample size estimate on results derived from studies investigating the effect of aerobic exercise training on HRV in apparently healthy adults [38]. The experimental group was expected to increase the HF component of HRV following the 16-week yoga intervention $\left(568.0 \pm 696.0 \mathrm{~ms}^{2}\right)$ and the control group was expected to experience no change $\left(205.7 \pm 290.5 \mathrm{~ms}^{2}\right)$. With a one-sided alpha level of 0.05 , at least 56 participants (28 per group) were required to provide $80 \%$ power to detect a statistically significant difference between groups. Recruitment was inflated to 68 participants to enable a $15 \%$ participant attrition rate.

\section{Randomization}

Participants were randomized via a computer-generated list (www.randomization.com) stratified by sex and age ( $<50$ yr.; $\geq 50$ yr). An investigator not involved in testing or the delivery of the intervention prepared the randomization assignments. Group assignments were delivered to participants in person in sealed envelopes upon the completion of baseline testing.

\section{Interventions}

\section{Experimental group}

Participants in the experimental group engaged in 16weeks of Bikram yoga classes (Fig. 1) at either of two affiliated Bikram yoga studios in Canberra, ACT. Participants were instructed to attend between three to five regularly scheduled classes per week. Certified Bikram yoga teachers instructed all classes using a set instructional dialogue. Classes were $90 \mathrm{~min}$ in duration and held in a temperature-controlled room $\left(40{ }^{\circ} \mathrm{C}, 40 \%\right.$ relative humidity). The Bikram yoga practice consisted of 45-50 min of standing asanas starting with a deep breathing exercise, and 40-45 min of floor-based asanas, including a quick, forceful breathing exercise to finish. All but the last asana (spine-twisting) were performed twice. Savasana (a restorative, relaxation posture) was performed between asanas in the floor series and at the end of class [26].

\section{Control group}

Participants in the control group were instructed to maintain current lifestyle practices and were not

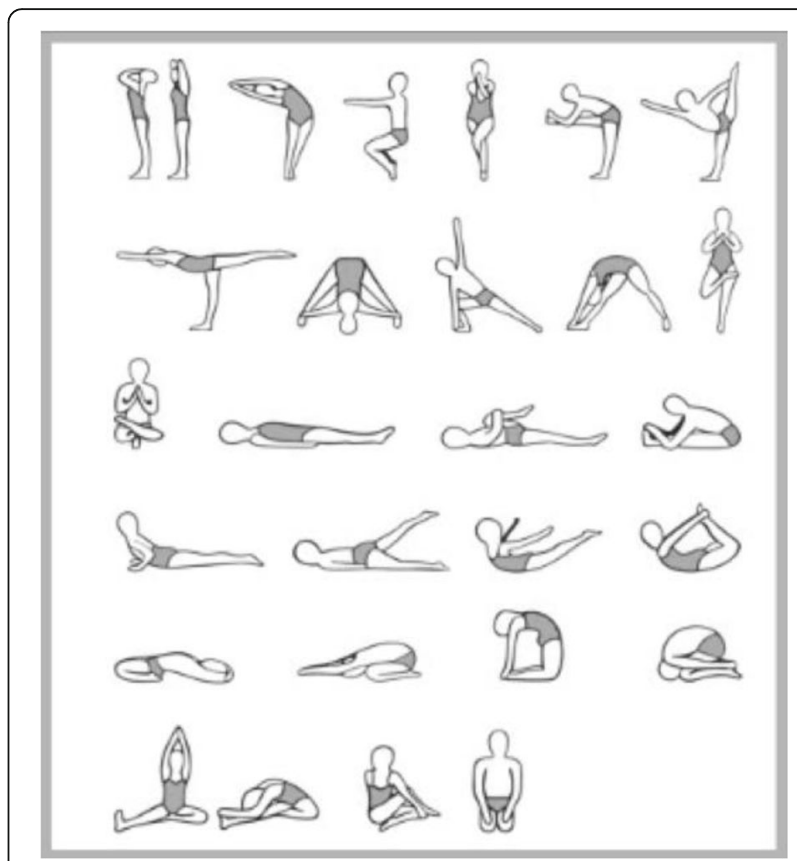

Fig. 1 Bikram yoga sequence of asanas (left to right, top to bottom)

provided any information or instructions about Bikram yoga practice. However, participants were informed during recruitment and screening that the control group participants would be provided a complimentary 10class pass at one of the participating studios upon completion of the trial.

\section{Outcome measures}

Two trained researchers collected all outcome measures at the University of Canberra, with the exception of hematological data, which were collected and analyzed on a separate day by Capital Pathology, a National Association of Testing Authorities (NATA) certified blood collection center (blinded). Participants were instructed to fast overnight $(12 \mathrm{~h})$ and refrain from exercise for $48 \mathrm{~h}$ prior to both the two testing session appointment and the blood test appointment. Appointments were rescheduled if these instructions were not followed. Follow up testing was scheduled at the same time of day as baseline testing to minimize the effect of diurnal variation.

\section{Heart rate variability outcomes}

HRV was measured in a quiet room according to guidelines developed by the Task Force for Pacing and Electrophysiology [14]. After $10 \mathrm{~min}$ of supine rest with a regular and calm breathing pattern, a continuous 10min ECG recording was collected using the SphygmoCor system and HRV software (SphygmoCor, AtCor Medical Pty, Sydney, Australia). From the ECG recording, the following time domain variables were 
calculated from r-wave to r-wave (RR) intervals: standard deviation of normal-to-normal (NN) intervals (SDNN), root mean square of successive differences between adjacent NN intervals (RMSSD), the proportion of the number of pairs of successive NN intervals that differ by $50 \mathrm{~ms}$ divided by the total $\mathrm{NN}$ intervals (pNN50) and HRV Triangular Index. Frequency domain variables, including HF and LF power (absolute), LF:HF and total power (TP) were derived from spectral analysis of successive RR intervals. The primary outcome of the present study was the HF spectral power component of HRV (measured in absolute units; $\mathrm{ms}^{2}$ ), while the other HRV measures were collected as secondary outcomes.

\section{Hemodynamic outcomes}

Resting brachial blood pressure (systolic and diastolic) and heart rate were assessed after lying for $10 \mathrm{~min}$ in a supine position using an automatic monitor (M10-IT, Omron Inc., Japan). Augmentation index (AIx) was then measured in this position at the radial artery using the SphygmoCor System (AtCor Medical Pty, Sydney, Australia) and hand-held, high fidelity tonometer (Millar Instruments, Houston, Texas). AIx data was normalized to a heart rate of 75 beats per minute to minimize the effects of confounding variables heart rate and ejection fraction [39].

\section{Hematological outcomes}

High-sensitivity c-reactive protein (hsCRP), triglycerides, total cholesterol, low-density- and high-density lipoprotein cholesterol (LDL and HDL), total cholesterol to HDL ratio (TC:HDL) and fasting blood glucose were collected and assessed using standard blood collection procedures and assays (coefficients of variation: 2.0\% $4.2 \%)$.

\section{Anthropometrics and body composition outcomes}

Height and weight were measured using a Seca 763 Electronic weighing and measuring scale (Seca, Hamburg, Germany) and BMI was computed from these measures [40]. Waist circumference was measured using a standardized procedure [40]. Body composition was assessed using a Lunar Prodigy Pro ${ }^{\text {tw }}$ Dual-energy X-ray Absorptiometry (DXA) scan analyzed with manufacturer software (enCORE $^{\text {ma }}$ v 14.1 software, GE Healthcare, Sydney, Australia). DXA scanning was completed in accordance with the University of Canberra's DXA scanning protocol and has been validated in previous research $[41,42]$. Participants were scanned prior to any exercise being completed and wore minimal clothing with all jewelry and metal objects removed. Data from participants too large to fit within the scanning region was estimated using the software estimate function. Percent body fat
(\%), fat mass (g), lean mass (g), fat mass (g), and bone mineral content $(\mathrm{g})$ were reported for all participants.

\section{Health status covariates, attendance and adverse events} Weekly status checks administered via phone, email or in person throughout intervention period were used to check for major exercise or diet changes and adverse events in the experimental and control group. A 7-day food diary was also completed in weeks 0 and 17 and analyzed using FoodWorks (version 8, Xyris Software Pty Ltd., Australia) to assess changes in diet. Attendance in the experimental group was recorded electronically upon arrival at each respective studio via an online booking system controlled by the staff member at reception. Attendance was reported as total number of sessions completed. Adverse events were defined as any injury directly attributable to the Bikram yoga intervention. Participants who experienced an adverse event were advised to visit a qualified health care practitioner for assessment and treatment.

\section{Statistical analyses}

Primary analysis was undertaken using intention-to-treat regardless of dropout or level of adherence. Missing data at week 17 was imputed using the last observation carry forward method. Outcomes data is presented as the mean \pm standard deviation (SD) with effect size and 95\% confidence intervals (CIs). Baseline characteristics were compared using t-tests (continuous variables) and chi square tests (categorical variables). Natural logarithm transformations were applied to variables showing positive skew, and accepted where normality was improved. Mean differences in outcomes between groups at completion were examined using analysis of covariance (ANCOVA) adjusting for the baseline value of the outcome variable. Pearson's correlation coefficients $(r)$ were used to examine baseline associations between the primary outcome and secondary outcomes with $p$-values reported for the hypothesis testing that the correlation was equal to zero. Regression analyses were used to examine the effect of attendance on outcomes. A $p$ value less than 0.05 was considered indicative of statistical significance with $p$ values less than 0.10 providing weaker evidence of association. Effect sizes are summarised as partial eta-squared statistics. All analyses were carried out using SPSS (IBM@), Version 23).

\section{Results}

\section{Participants}

Two-hundred and twelve individuals contacted the principal investigator and were reviewed for eligibility; 139 were deemed ineligible for reasons presented in Fig. 2. Of 73 eligible individuals, 68 provided written informed consent and were randomized to either the experimental 


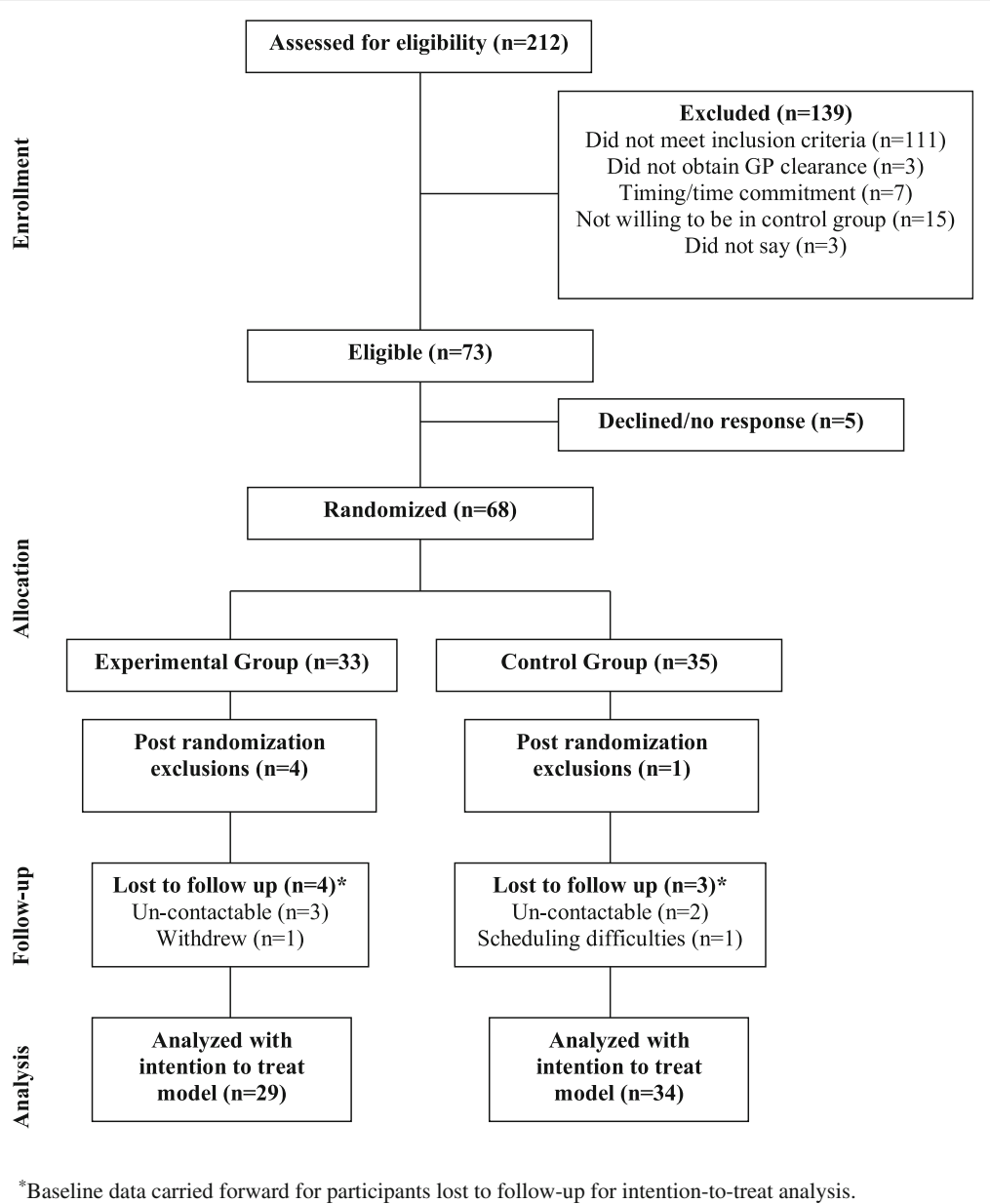

Fig. 2 Participant flow

or control group. Four participants in the experimental group and one participant from the control group were excluded from analysis post-randomization after recalculation of the DASS-21 stress scores (inclusion criterion score not met). Further, four participants in the experimental group and three participants in the control group did not return for follow-up testing (week 17).

\section{Baseline characteristics}

At baseline there were no significant differences between groups in any of the descriptive characteristics presented in Table 1. Participants ranged from 19 to 64 years of age. The majority of the cohort $(87 \%)$ was under 50 years of age (55/63) and $79 \%$ of the cohort was comprised of women (50/63). Further, $43 \%$ of the

Table 1 Baseline characteristics

\begin{tabular}{|c|c|c|c|}
\hline Characteristic & $\begin{array}{l}\text { Total cohort } \\
(n=63)\end{array}$ & $\begin{array}{l}\text { Yoga group } \\
(n=29)\end{array}$ & $\begin{array}{l}\text { Control group } \\
(n=34)\end{array}$ \\
\hline Age (y) & $37.2 \pm 10.8$ & $38.2 \pm 10.1$ & $36.3 \pm 11.4$ \\
\hline Women (n; \%) & $50 ; 79 \%$ & $23 ; 79 \%$ & $27 ; 79 \%$ \\
\hline Body weight (kg) & $88.5 \pm 21.0$ & $86.4 \pm 21.2$ & $90.4 \pm 21.0$ \\
\hline Body mass index (BMI) $\left(\mathrm{kg} / \mathrm{m}^{2}\right)$ & $30.5 \pm 6.2$ & $29.9 \pm 6.2$ & $30.9 \pm 6.3$ \\
\hline Obese (BMI 30+) (n; \%) & $27 ; 43 \%$ & $11 ; 38 \%$ & $16 ; 47 \%$ \\
\hline Waist circumference (cm) & $94.7 \pm 14.7$ & $93.9 \pm 14.5$ & $95.3 \pm 15.1$ \\
\hline Stress score (DASS-21) & $23.8 \pm 6.0$ & $23.3 \pm 6.0$ & $24.3 \pm 6.1$ \\
\hline Current smoker or quit in last 6 months (n; \%) & $3 ; 5 \%$ & $1 ; 3 \%$ & $2 ; 6 \%$ \\
\hline
\end{tabular}

Continuous data presented as mean \pm standard deviation

Abbreviations: DASS Depression-Anxiety-Stress-Scale (stress categories: $15-18=$ mild, $19-25=$ moderate, $26-33=$ severe, $34+=$ extremely severe) 
cohort (27/63) meet the clinical criteria for obesity according to BMI $>30 \mathrm{~kg} / \mathrm{m}^{2}$. The average DASS-21 stress score for the total cohort indicated a moderate level of stress $(23.8 \pm 6.0)$. Nearly $70 \%$ of the total cohort had no prior Bikram yoga experience while $90 \%$ had engaged in less than five Bikram yoga classes previously.

\section{Health status covariates, attendance and adverse events}

There were no significant changes between groups over time in total energy, carbohydrate, protein and fat intake according to the 7-day food diary. Weekly status checks revealed that three participants in the control group engaged in structured exercise programs during the intervention period (i.e. aerobic and/or strength training).

The experimental group attended an average of $27 \pm 18$ (range: 4 to 79 ) of a possible 80 classes. Nine participants attended $>80 \%$ of the minimum three required classes per week (i.e. $>38$ of 48 classes). Ten participants attended a total of 16 or fewer classes (i.e. less than one class per week) over the intervention period.

Six participants in the experimental group experienced exacerbation of a pre-existing condition during the trial (i.e. back pain, foot pain, knee pain, calf pain, psychological discomfort). All six participants were advised to consult their general practitioner. Three of these participants discontinued the intervention. The other three continued attending some classes when they could, with modifications made to asanas if needed. One additional participant discontinued the intervention after a nonintervention related event (sprained ankle). In the control group, one participant reported a meniscus tear incurred by stepping awkwardly.

\section{Baseline associations with the primary outcome}

After logarithmic transformation (Ln) to correct skewed distributions, the Ln HF power component of HRV was found to be positively correlated with all secondary HRV outcomes (all $r>0.6$, all $p<0.001$ ). Further, higher Ln HF power was associated with lower age $(r=-0.44$, $p<0.001)$, resting heart rate $(r=-0.47, p<0.001)$, systolic blood pressure $(r=-0.33, p=0.008)$, diastolic blood pressure $(r=-0.27, p=0.031)$, total cholesterol $(r=-0.26, p=0.038)$, LDL cholesterol $(r=-0.26$, $p=0.038)$, and TC:HDL $(r=-0.33, p=0.008)$.

\section{Outcomes}

All outcomes are presented in Table 2.

\section{Heart rate variability}

No significant difference in the Ln HF power component of HRV was observed between groups over time $\left(p=0.912\right.$, partial $\left.\eta^{2}=0.000\right)$. Further, no group by time interactions were noted in any of the secondary HRV outcomes, including Ln LF power $(p=0.424$, partial $\left.\eta^{2}=0.011\right)$, Ln LF:HF $\left(p=0.978\right.$, partial $\left.\eta^{2}=0.000\right)$, Ln total power $\left(p=0.755\right.$, partial $\left.\eta^{2}=0.002\right)$, pNN50 $\left(p=0.353\right.$, partial $\left.\eta^{2}=0.014\right)$, Ln SDNN $(p=0.948$, partial $\left.\eta^{2}=0.000\right)$, Ln RMSSD $(p=0.942$, partial $\left.\eta^{2}=0.000\right)$ and Ln Triangular Index $(p=0.486$, partial $\left.\eta^{2}=0.008\right)$.

\section{Hemodynamic outcomes}

No differences between groups over time were noted for resting heart rate $\left(p=0.248\right.$, partial $\left.\eta^{2}=0.022\right)$, systolic blood pressure $\left(p=0.517\right.$, partial $\left.\eta^{2}=0.007\right)$, diastolic blood pressure $\left(p=0.769\right.$, partial $\left.\eta^{2}=0.001\right)$ or AIx $\left(p=0.294\right.$, partial $\left.\eta^{2}=0.022\right)$.

\section{Hematological outcomes}

No differences between groups over time were found in total cholesterol $\left(p=0.635\right.$, partial $\left.\eta^{2}=0.004\right)$, HDL $\left(p=0.886\right.$, partial $\left.\eta^{2}=0.000\right)$, TC:HDL $(p=0.450$, partial $\left.\eta^{2}=0.010\right)$, LDL $\left(p=0.412\right.$, partial $\left.\eta^{2}=0.011\right)$, triglycerides $\left(p=0.522\right.$, partial $\left.\eta^{2}=0.007\right)$, fasting blood glucose $\left(p=0.672\right.$, partial $\left.\eta^{2}=0.003\right)$ or Ln hsCRP $\left(p=0.430\right.$, partial $\left.\eta^{2}=0.010\right)$.

\section{Anthropometric and body composition outcomes}

No differences between groups over time were found in body weight $\left(p=0.618\right.$, partial $\left.\eta^{2}=0.004\right)$, BMI $\left(p=0.496\right.$, partial $\left.\eta^{2}=0.008\right)$ or waist circumference $\left(p=0.204\right.$, partial $\left.\eta^{2}=0.027\right)$. There were also no group by time interactions noted for body fat percentage $\left(p=0.231\right.$, partial $\left.\eta^{2}=0.024\right)$, fat mass $(p=0.714$, partial $\left.\eta^{2}=0.002\right)$, lean body mass $(p=0.126$, partial $\left.\eta^{2}=0.039\right)$, or fat free mass $(p=0.147$, partial $\left.\eta^{2}=0.035\right)$. The data of six participants who did not fit comfortably within the scan region were calculated using the software estimate function.

\section{Effect of adherence on adaptation}

ANCOVAs indicated that higher attendance in the experimental group (i.e. number of classes) was associated with significant reductions in diastolic blood pressure $\left(p=0.039\right.$, partial $\left.\eta^{2}=0.154\right)$, body fat percentage $\left(p=0.001\right.$, partial $\left.\eta^{2}=0.379\right)$, fat mass $(p=0.003$, partial $\left.\eta^{2}=0.294\right)$ and BMI $\left(p=0.05\right.$, partial $\left.\eta^{2}=0.139\right)$. Moreover, weak evidence of association was noted between higher attendance and reduced systolic blood pressure $\left(p=0.072\right.$, partial $\left.\eta^{2}=0.119\right)$, body weight $\left(p=0.062\right.$, partial $\left.\eta^{2}=0.128\right)$, waist circumference $\left(p=0.072\right.$, partial $\left.\eta^{2}=0.120\right)$ and HDL cholesterol $\left(p=0.052\right.$, partial $\left.\eta^{2}=0.137\right)$.

\section{Discussion}

The present study investigated the effect of 16-weeks of Bikram yoga on the HF component of HRV, and secondary CVD risk factors in sedentary, stressed adults. 
Table 2 Summary of between group changes on clinical outcomes

\begin{tabular}{|c|c|c|c|c|c|c|c|}
\hline \multirow[t]{2}{*}{ Outcome Measure } & \multicolumn{2}{|l|}{ Yoga $(n=29)$} & \multicolumn{2}{|l|}{ Control $(n=34)$} & \multirow{2}{*}{$\begin{array}{l}\text { Unadjusted Mean } \\
\text { difference ( } 95 \% \text { Cl) }\end{array}$} & \multirow{2}{*}{$\begin{array}{l}P \\
\text { (between } \\
\text { groups) }\end{array}$} & \multirow{2}{*}{$\begin{array}{l}\text { Effect Size } \\
\left(\text { partial } \eta^{2}\right.\end{array}$} \\
\hline & Week 0 & Week 17 & Week 0 & Week 17 & & & \\
\hline \multicolumn{8}{|l|}{ Heart Rate Variability } \\
\hline Ln HF power (absolute) & $6.39 \pm 1.30$ & $6.27 \pm 1.30$ & $5.67 \pm 1.46$ & $5.76 \pm 1.48$ & $-0.503(-1.203-0.196)$ & 0.912 & 0.000 \\
\hline Ln LF power (absolute) & $6.14 \pm 1.17$ & $5.93 \pm 1.09$ & $5.66 \pm 1.10$ & $5.44 \pm 1.19$ & $-0.488(-1.063-0.088)$ & 0.424 & 0.011 \\
\hline Ln LF:HF ratio & $-0.244 \pm 0.95$ & $-0.337 \pm 0.92$ & $-0.002 \pm 1.17$ & $-0.213 \pm 1.17$ & $0.124(-0.406-0.653)$ & 0.978 & 0.000 \\
\hline Ln total power & $7.52 \pm 1.01$ & $7.33 \pm 1.05$ & $7.02 \pm 0.94$ & $6.92 \pm 1.06$ & $-0.406(-0.940-0.128)$ & 0.755 & 0.002 \\
\hline pNN50 & $21.81 \pm 21.35$ & $21.46 \pm 22.71$ & $18.72 \pm 20.74$ & $21.80 \pm 24.49$ & $0.338(-11.566-12.243)$ & 0.353 & 0.014 \\
\hline Ln SDNN & $3.99 \pm 0.46$ & $3.95 \pm 0.48$ & $3.74 \pm 0.44$ & $3.77 \pm 0.50$ & $-0.183(-0.429-0.063)$ & 0.948 & 0.000 \\
\hline Ln RMSSD & $3.78 \pm 0.65$ & $3.80 \pm 0.68$ & $3.50 \pm 0.68$ & $3.61 \pm 0.68$ & $-0.188(-0.531-0.156)$ & 0.942 & 0.000 \\
\hline Ln Triangular Index & $5.91 \pm 0.45$ & $5.92 \pm 0.43$ & $5.85 \pm 0.48$ & $5.83 \pm 0.51$ & $-0.098(-0.333-0.138)$ & 0.486 & 0.008 \\
\hline \multicolumn{8}{|l|}{ Hemodynamic measures } \\
\hline Resting heart rate (bpm) & $64.1 \pm 8.0$ & $62.9 \pm 8.3$ & $65.4 \pm 9.1$ & $62.2 \pm 8.7$ & $-0.784(-5.084-3.516)$ & 0.248 & 0.022 \\
\hline Systolic blood pressure $(\mathrm{mmHg})$ & $120.3 \pm 11.1$ & $119.1 \pm 10.3$ & $119.8 \pm 9.1$ & $119.6 \pm 8.9$ & $0.49(-4.419-5.399)$ & 0.517 & 0.007 \\
\hline Diastolic blood pressure $(\mathrm{mmHg})$ & $74.7 \pm 7.9$ & $73.5 \pm 8.0$ & $76.1 \pm 6.4$ & $74.3 \pm 7.2$ & $0.875(-2.970-4.721)$ & 0.769 & 0.001 \\
\hline Augmentation index (@75 bpm) ${ }^{\mathrm{a}}$ & $16.1 \pm 9.8$ & $16.2 \pm 8.8$ & $12.9 \pm 13.1$ & $13.5 \pm 11.3$ & $-2.678(-7.842-2.487)$ & 0.294 & 0.022 \\
\hline \multicolumn{8}{|l|}{ Hematological measures } \\
\hline Total cholesterol (mmol/L) & $5.15 \pm 0.84$ & $5.15 \pm 0.69$ & $5.03 \pm 0.91$ & $4.98 \pm 1.11$ & $-0.169(-0.628-0.289)$ & 0.635 & 0.004 \\
\hline $\mathrm{HDL}(\mathrm{mmol} / \mathrm{L})$ & $1.59 \pm 0.52$ & $1.53 \pm 0.47$ & $1.40 \pm 0.38$ & $1.37 \pm 0.34$ & $-0.157(-0.369-0.055)$ & 0.886 & 0.000 \\
\hline TC/HDL ratio & $3.53 \pm 1.24$ & $3.65 \pm 1.20$ & $3.83 \pm 1.25$ & $3.84 \pm 1.26$ & $0.187(-0.435-0.808)$ & 0.450 & 0.010 \\
\hline $\mathrm{LDL}(\mathrm{mmol} / \mathrm{L})^{\mathrm{a}}$ & $2.94 \pm 0.75$ & $3.07 \pm 0.71$ & $3.03 \pm 0.84$ & $3.02 \pm 1.01$ & $-0.045(-0.481-0.390)$ & 0.412 & 0.011 \\
\hline Triglycerides (mmol/L) & $1.27 \pm 0.93$ & $1.22 \pm 0.64$ & $1.28 \pm 0.81$ & $1.28 \pm 0.65$ & $0.065(-0.260-0.391)$ & 0.522 & 0.007 \\
\hline Fasting blood glucose (mmol/L) & $5.01 \pm 0.58$ & $5.04 \pm 0.57$ & $4.94 \pm 0.44$ & $4.94 \pm 0.52$ & $-0.100(-0.377-0.177)$ & 0.672 & 0.003 \\
\hline Ln hsCRP & $0.64 \pm 1.35$ & $0.51 \pm 1.27$ & $0.80 \pm 1.23$ & $0.78 \pm 1.22$ & $0.268(-0.363-0.900)$ & 0.430 & 0.010 \\
\hline \multicolumn{8}{|l|}{ Body Composition ${ }^{a}$} \\
\hline Body weight (kg) & $86.4 \pm 21.2$ & $86.2 \pm 21.3$ & $90.4 \pm 21.0$ & $91.3 \pm 21.3$ & $4.328(-6.279-14.935)$ & 0.618 & 0.004 \\
\hline Body mass index $\left(\mathrm{kg} / \mathrm{m}^{2}\right)$ & $29.9 \pm 6.2$ & $29.8 \pm 6.2$ & $30.9 \pm 6.3$ & $31.0 \pm 6.3$ & $1.186(-1.977-4.349)$ & 0.496 & 0.008 \\
\hline Waist circumference (cm) & $93.9 \pm 14.5$ & $92.6 \pm 14.7$ & $95.3 \pm 15.1$ & $95.0 \pm 14.1$ & $2.399(-4.898-9.696)$ & 0.204 & 0.027 \\
\hline Fat mass (kg) & $34.5 \pm 14.5$ & $34.4 \pm 14.4$ & $38.0 \pm 14.0$ & $37.5 \pm 13.8$ & $3.141(-4.066-10.348)$ & 0.714 & 0.002 \\
\hline Lean mass (kg) & $48.8 \pm 10.4$ & $48.8 \pm 10.7$ & $48.3 \pm 11.0$ & $48.9 \pm 10.8$ & $0.117(-5.390-5.624)$ & 0.126 & 0.039 \\
\hline Fat-free mass (kg) & $51.7 \pm 10.9$ & $51.8 \pm 11.2$ & $51.4 \pm 11.4$ & $52.0 \pm 10.9$ & $0.150(-5.575-5.876)$ & 0.147 & 0.035 \\
\hline Body fat (\%) & $40.4 \pm 9.3$ & $40.4 \pm 9.3$ & $43.2 \pm 8.7$ & $42.6 \pm 8.8$ & $2.188(-2.442-6.819)$ & 0.231 & 0.024 \\
\hline
\end{tabular}

Data reported as mean (standard deviation). Abbreviations HF high frequency, LF low frequency, $p N N 50$ percentage of absolute differences between successive normal RR intervals that exceed $50 \mathrm{~ms}$, SDNN standard deviation of the normal-normal interval, RMSSD root-mean-square of the successive normal sinus RR interval difference, $H D L$ high-density lipoprotein, $T C / H D L$ ratio of total cholesterol to $H D L$, $L D L$ low-density lipoprotein, $h s C R P$ high sensitivity $c$-reactive protein * $=p<0.05$

${ }^{\mathrm{a}}=$ sample size differs to main data set. DEXA data: yoga group $n=28$. Alx: baseline yoga group $n=22$, baseline control group $n=30$, completion yoga group $n=27$. LDL: baseline yoga group $n=28$

Intention-to-treat analysis revealed that the Bikram yoga intervention did not significantly improve the HF power component of HRV or any other CVD risk factors investigated, contrary to our hypotheses. Attendance in the experimental group $(n=29)$ was low, averaging 27 classes, which is $56 \%$ of the minimum 48 classes required. Low attendance may have contributed to our null findings. Regression analyses revealed that higher attendance was associated with significant reductions in diastolic blood pressure, body fat percentage, fat mass, and BMI. Continued investigation into adherence to Bikram yoga interventions may reveal further information on the dose-response effect of Bikram yoga on CVD risk factors.

Our findings for HRV outcomes are supported by a recent systematic review [25], which concluded that current evidence cannot describe a definitive effect of chronic hatha yoga practice on measures of HRV, 
including HF power HRV, LF power HRV, LF:HF, TP, SDNN, and RMSSD. The HF component of HRV can be increased acutely by a reduced respiration rate, which likely contributed to the positive findings noted in some acute trials [25, 43]. The present study suggests that there is no chronic increase in vagal tone (relaxation response) in response to the intervention, however previous research suggests that Bikram and non-Bikram yoga interventions do reduce psychological stress $[16,28]$ and reduce cortisol reactivity to stress [27]. Heart rate variability can also be increased from via exercise-induced adaptation of the cardiovascular system. Research to date indicates that Bikram yoga does not elicit a strong enough cardiovascular training load in sedentary, apparently healthy adults to improve maximal oxygen update $[29,44]$. The null findings in the present study may be due to the lack of chronic adaptation of the cardiac muscle (i.e. increased stroke volume) required for adaptation in HRV despite past evidence for reduced psychological stress from Bikram yoga intervention. Preliminary data show that Bikram yoga elicits an average, in-session metabolic response equivalent to that of walking $[45,46]$ which may, in a higher risk, less fit population, lead to increased HRV via adaptation in cardiac muscle and stroke volume after a Bikram yoga intervention. Interestingly, a study examining integrated hatha yoga (ethical and spiritual components) versus hatha yoga (as exercise only) reports that only those in the integrated group experienced significant decreases in physiological measures of stress (i.e. salivary cortisol) [47]. This finding suggests that the framework of hatha yoga RCTs, including the reasons for which participants initially register, may influence adaptations of physiological stress markers.

There was a null effect of Bikram yoga on secondary hemodynamic outcomes in the present study. Our findings in this cohort of normotensive adults (Table 2) support those of previous studies (controlled and uncontrolled), which report unchanged blood pressure and resting heart rate in normotensive adults after 8 weeks of Bikram or non-Bikram hatha yoga performed one to three sessions per week [24, 28, 29, 44]. Interestingly, a cross-sectional study reported that resting blood pressure of long-term Bikram yoga practitioners (1 year) was lower than the general United States population indicating that this practice may have a beneficial effect on hemodynamic health [48]. Moreover, longer (5-6 months) hatha yoga RCTs report significant improvements in blood pressure in coronary artery disease patients and pre-hypertensive individuals living with HIV infection [49-51]. A previous study in healthy young adults has also noted within group reductions in arterial stiffness measured at the carotid artery after 8 weeks of Bikram yoga intervention [29].
The significant association between adherence and diastolic blood pressure $\left(p=0.039\right.$, partial $\left.\eta^{2}=0.154\right)$, and weaker evidence of association in systolic blood pressure $\left(p=0.072\right.$, partial $\left.\eta^{2}=0.119\right)$ in the present study indicate that there may be a dose-response effect for blood pressure changes in relation to Bikram yoga. Considering both the relationship between adherence and adaptation, and the potential vascular benefits of heat therapy shown in several studies [31, 52, 53], an $\mathrm{RCT}$ in a pre-hypertensive or hypertensive cohort, with adequate adherence to intervention, may elicit Bikram yoga intervention-related changes in systolic and diastolic blood pressure.

No significant changes were found for hematological CVD risk factors. An 8-week, uncontrolled Bikram trial also reported no change to fasting blood glucose in adults, although improvements in glucose tolerance were noted in obese adults [29]. Contrary to the present results, however, an uncontrolled 8-week Bikram yoga trial reported significant within group decreases in TC and LDL in older adults, and significant within group decreases in TC and HDL in young adults [29]. The current trial also provides weak evidence for reduced HDL with higher attendance $(p=0.052$, partial $\left.\eta^{2}=0.137\right)$. This is an unexpected HDL change in response to exercise, however, HDL levels were $1.53 \pm 0.47 \mathrm{mmol} / \mathrm{L}$ at completion, which is still well above recommended levels for a healthy blood lipid profile $(>1.0 \mathrm{mmol} / \mathrm{L})$. The same 8 -week study also reports that plasma insulin and insulin resistance via homeostatic model assessment (HOMA-IR) improved significantly (within group) in older adults [29]. Further, RCTs longer than 8 weeks suggest that hatha yoga can improve blood lipids, blood glucose and hemoglobin A1c in unhealthy populations $[49,50,54]$. There was no adaptation in hsCRP in the present study. This finding is contrary to a 4-week hatha yoga RCT in apparently healthy, male railway engine drivers, and an 8-week hatha yoga RCT in heart failure patients $[55,56]$. Although adherence did not appear to be associated with blood measure outcomes, it is possible that a higher risk cohort with elevated CVD risk factor blood measures at baseline would experience significant reductions in response to an appropriate volume of Bikram yoga training.

There was no significant adaptation of body weight, body composition, waist circumference or BMI in the present study. An RCT reported a trend in reduced body fat after 8-weeks of Bikram yoga, although no diet data was recorded during that 8-week trial [44]. Preliminary energy expenditure data from two studies indicates that Bikram yoga elicits a higher metabolic equivalent (MET) energy expenditure level compared to other forms of hatha yoga, and that it can be compared energetically to walking up to 3.7 METS [45, 46, 57]. Both studies 
include data from experienced practitioners, who may have higher exertion and energy expenditure rates during class than that of the current, less experienced, less active practitioners. Yoga has been associated with attenuated weight gain in healthy adults over a 10 -year period [58]. In the current trial, more regular attendance was associated with reductions in body fat percentage $\left(p=0.001\right.$, partial $\left.\eta^{2}=0.379\right)$, fat mass $(p=0.003$, partial $\left.\eta^{2}=0.294\right)$ and BMI $\left(p=0.05\right.$, partial $\left.\eta^{2}=0.139\right)$. These data suggest that Bikram yoga may serve as an effective weight maintenance tool and, with an appropriate training volume, a tool for body fat reduction.

A major strength of this study was its rigorous RCT design [59], which is currently lacking in the investigation of Bikram yoga. A major limitation to the study was low attendance with participants attending on average 1.7 classes per week compared to the minimum requirement of three classes per week. Poor attendance could be due to the duration of the 16-week trial or the time demand of the 90-min classes. Another limitation to the study is that the cohort may have been too low-risk to see adaptation in certain measures including blood pressure, blood lipids and hsCRP. Further, data was collected without considering the timing in relation to menstrual cycle. In this predominantly female cohort, that oversight may have affected some outcome measures. Some participants experienced discomfort relating to pre-existing musculoskeletal conditions during the study, which would not be uncommon when starting an exercise program after being sedentary for some period. The intervention may have exacerbated this discomfort for some participants due to the group exercise setting. To reduce intervention-related discomfort, future study design could include one pre-intervention yoga class to better familiarize participants with the protocol and to ensure that they are given specific, individual feedback for performing the asanas in a way that minimizes discomfort.

Future research should explore the effects of Bikram yoga in unhealthy populations to better examine the effects of this form of heated hatha yoga on CVD risk factors. However, in light of the association of adherence on the outcomes demonstrated in this study, more acute and intervention-based research is required to determine a suitable, minimum volume of Bikram yoga in order to encourage an adequate level of adherence that elicits physiological change in various outcomes. Furthermore, cross-sectional and longitudinal data on long term practitioners could lend valuable insight into the characteristics of a Bikram yoga practitioner, including motivation to start and continue a Bikram yoga practice, as well as perceived barriers to continue. Determining how to better facilitate adherence to Bikram yoga interventions is an important consideration moving forward, especially considering that a stressed cohort with little yoga experience potentially faces more barriers to initiating a yoga practice than an individual initiating a yoga practice independent of the RCT framework.

\section{Conclusion}

In summary, a 16-week Bikram yoga program did not increase the HF power component of HRV or any other CVD risk factors investigated. Low adherence likely contributed to the null effects. Regression analyses indicated that higher attendance was significantly associated with reductions in diastolic blood pressure, body fat, fat mass and BMI, and weaker associations were noted in systolic blood pressure, body weight and waist circumference. Future studies are required to address barriers to adherence and elucidate the dose-response effects of Bikram yoga practice.

\section{Abbreviations \\ ACT: Australian capital territory; Alx: Augmentation index; ANCOVA: Analysis of covariance; ANS: Autonomic nervous system; BMI: Body mass index; $\mathrm{Cl}$ : Confidence interval; CVD: Cardiovascular disease; DASS-21: 21-item depression-anxiety-stress scale; DXA: Dual-energy x-ray absorptiometry; ECG: Electrocardiogram; HF: High frequency; HDL: High-density lipoprotein; HPA: Hypothalamic-pituitary-adrenal; HRV: Heart rate variability; hsCRP: High- sensitivity c-reactive protein; LF: Low frequency; LDL: Low-density lipoprotein Ln: Natural logarithm; MET: Metabolic equivalent; NATA: National Association of Testing Authorities; NN: Normal-to-normal; PAR-Q: Physical activity readiness questionnaire; pNN50: proportion of the number of pairs of successive NN intervals that differ by 50 ms divided by the total NN intervals; $\mathrm{RCT}$ : Randomized controlled trial; RMSSD: Root mean square of successive differences between adjacent NN intervals; RR: r-wave to r-wave; SD: Standard deviation; SDNN: Standard deviation of NN intervals; SNS: Sympathetic nervous system; SPSS: Statistical package for the social sciences; TC: Total cholesterol; TP: Total power}

\section{Acknowledgements}

Thank you to Dr. Jocelyn Mara who provided supplementary statistical guidance in Canberra. Thank you to Bikram Yoga Canberra and Bikram Yoga Kingston for providing access to the yoga classes free of charge.

\section{Funding}

This research was supported by PhD research funding provided by Western Sydney University. Bikram Yoga Canberra and Bikram Yoga Kingston allowed participants to attend classes free of charge and offered 10 complimentary classes (value \$180) to control group participants.

\section{Availability of data and material}

The datasets used and/or analyzed during the current study available from the corresponding author on reasonable request.

\section{Authors' contributions}

Conceived the study: ZLH. Designed the study: ZLH, BSC, KLP, CS. Acquisition of data: ZLH, KLP. Delivered intervention: ZLH (and primarily other instructors at Bikram Yoga Canberra and Bikram Yoga Kingston). Prepared manuscript: ZLH, BSC, KLP, PF, CS. Statistical analysis guidance: PF. All authors have read and approved the final manuscript.

\section{Competing interests}

ZLH declares that she became a co-owner of Bikram Yoga Kingston in July 2014, after the conception and design of the study. All other authors declare they have no competing interests.

Consent for publication

Not applicable. 


\section{Ethics approval and consent to participate}

The Western Sydney University (H10549) and University of Canberra Human Research Ethics Committees (H10549-14/009174) approved all procedures and written informed consent was obtained from all participants.

\section{Publisher's Note}

Springer Nature remains neutral with regard to jurisdictional claims in published maps and institutional affiliations.

\section{Author details}

${ }^{1}$ School of Science and Health, Western Sydney University, Locked Bag 1797, Penrith, NSW 2751, Australia. ${ }^{2}$ National Institute of Complementary Medicine, Western Sydney University, Penrith, NSW 2751, Australia. ${ }^{3}$ Research Institute for Sport and Exercise, University of Canberra, ACT, Canberra 2617, Australia.

Received: 6 December 2016 Accepted: 12 April 2017

Published online: 21 April 2017

\section{References}

1. Thayer JF, Lane RD. The role of vagal function in the risk for cardiovascular disease and mortality. Biol Psychol. 2007;74(2):224-42.

2. Foss B, Dyrstad SM. Stress in obesity: Cause or consequence? Med Hypotheses. 2011;77(1):7-10.

3. Dishman RK, et al. Heart rate variability, trait anxiety, and perceived stress among physicall fit men and women. Int J Psychophysiol. 2000;37:121-33.

4. Bose M, Oliván B, Laferrère B. Stress and obesity: the role of the hypothalamic-pituitary-adrenal axis in metabolic disease. Curr. Opin. Endocrinol. Diabetes Obes. 2009;16(5):340-6.

5. Charmandari E, Tsigos C, Chrousos G. Endocrinology of the stress response 1. Annu Rev Physiol. 2005;67:259-84.

6. Chandola T, et al. Work stress and coronary heart disease: what are the mechanisms? Eur Heart J. 2008;29(5):640-8.

7. Chrousos GP. The role of stress and the hypothalamic-pituitary-adrenal axis in the pathogenesis of the metabolic syndrome: neuro-endocrine and target tissue-related causes. Int. J. Obes. Relat. Metab. Disord. 2000;24:S50-5.

8. Ryan $\mathrm{ML}$, et al. Heart rate variability is an independent predictor of morbidity and mortality in hemodynamically stable trauma patients. J. Trauma Acute Care Surg. 2011;70(6):1371-80.

9. Thayer JF, Lane RD. Claude Bernard and the heart-brain connection: Further elaboration of a model of neurovisceral integration. Neurosci Biobehav Rev. 2009;33(2):81-8.

10. Nolan J, et al. Prospective study of heart rate variability and mortality in chronic heart failure results of the United Kingdom heart failure evaluation and assessment of risk trial (UK-Heart). Circulation. 1998:98(15):1510-6.

11. Dietrich DF, et al. Effect of physical activity on heart rate variability in normal weight, overweight and obese subjects: results from the SAPALDIA study. Eur J Appl Physiol. 2008;104(3):557-65.

12. Lampert $R$, et al. Decreased heart rate variability is associated with higher levels of inflammation in middle-aged men. Am Heart J. 2008;156(4):759.e1-7.

13. Andrew ME, et al. Adiposity, muscle, and physical activity: Predictors of perturbations in heart rate variability. Am J Hum Biol. 2013;25(3):370-7.

14. Task Force of the European Society of Cardiology the North American Society of Pacing Electrophysiology. Heart Rate Variability: Standards of Measurement, Physiological Interpretation, and Clinical Use. Circulation. 1996:93(5):1043-65.

15. Borg-Olivier S, Machliss B. Applied Anatomy and Physiology of Yoga. Waverly: Yoga Synergy; 2011

16. Chong CS, et al. Effects of yoga on stress management in healthy adults: A systematic review. Altern. Ther. Health Med. 2011;17(1):32-8.

17. Rocha KK, et al. Improvement in physiological and psychological parameters after 6 months of yoga practice. Conscious Cogn. 2012:21(2):843-50.

18. Banasik J, et al. Effect of lyengar yoga practice on fatigue and diurnal salivary cortisol concentration in breast cancer survivors. J Am Acad Nurse Pract. 2011;23(3):135-42.

19. West J, et al. Effects of Hatha yoga and African dance on perceived stress, affect, and salivary cortisol. Ann Behav Med. 2004;28(2):114-8.

20. Chu $P$, et al. The effectiveness of yoga in modifying risk factors for cardiovascular disease and metabolic syndrome: A systematic review and meta-analysis of randomized controlled trials. Eur J Prev Cardiol. 2014:291-307.

21. Huang F-J, Chien D-K, Chung U-L. Effects of Hatha yoga on stress in middleaged women. J Nurs Res. 2013;21(1):59-66.
22. Satyapriya $M$, et al. Effect of integrated yoga on stress and heart rate variability in pregnant women. Int J Gynecol Obstet. 2009;104(3):218-22.

23. Sawane MV, Gupta SS. Resting heart rate variability after yogic training and swimming: A prospective randomized comparative trial. Int J Yoga. 2015; 8(2):96-102.

24. Papp ME, et al. Increased heart rate variability but no effect on blood pressure from 8 weeks of hatha yoga - a pilot study. BMC. Res. Notes. 2013; 6:59-67.

25. Posadzki P, et al. Yoga for Heart Rate Variability: A Systematic Review and Meta-analysis of Randomized Clinical Trials. Appl. Psychophysiol. Biofeedback. 2015:40(3):239-49.

26. Choudhury B. Bikram Yoga. New York: Harper Collins; 2007.

27. Hopkins $L B$, et al. Heated hatha yoga to target cortisol reactivity to stress and affective eating in women at risk for obesity-related illnesses: A randomized controlled trial. J Consult Clin Psychol. 2016:84(6):558-64.

28. Hewett ZL, et al. An Examination of the Effectiveness of an 8-week Bikram Yoga Program on Mindfulness, Perceived Stress, and Physical Fitness. J. Exerc. Sci. Fit. 2011;9(2):87-92.

29. Hunter SD, et al. The Effect of Bikram Yoga on Arterial Stiffness in Young and Older Adults. J. Altern. Complement. Med. 2013;19(12):930-4.

30. Hunter SD, et al. Improvements in glucose tolerance with Bikram yoga in older obese adults: A pilot study. J Bodyw Mov Ther. 2013;17(4):404-7.

31. Laukkanen T, et al. Association between sauna bathing and fatal cardiovascular and all-cause mortality events. JAMA Intern Med. 2015;175(4):542-8.

32. Stanley J, et al. Effect of sauna-based heat acclimation on plasma volume and heart rate variability. Eur J Appl Physiol. 2015;115(4):785-94.

33. Corsini A, Capogrosso M, Perini R. Effects of spa treatment on cardiac autonomic control at rest in healthy subjects. Sport Sciences for Health. 2015;11(2):181-6.

34. Hewett ZL, et al. The Effects of Bikram Yoga on Health: Critical Review and Clinical Trial Recommendations. Evid Based Complement Alternat Med. 2015;2015:13.

35. ACSM. ACSM's guidelines for exercise testing and prescription. 7th ed. Philadelphia: Lippincott Williams \& Wilkins; 2006.

36. Lovibond PF, Lovibond SH. The structure of negative emotional states: Comparison of the Depression Anxiety Stress Scales (DASS) with the Beck Depression and Anxiety Inventories. Behav Res Ther. 1995;33(3):335-43.

37. Canadian Society for Exercise Physiology. Physical Activity Readiness Questionnaire (PAR-Q). 2002 [cited 201618 August]; Available from: http:// www.csep.ca/CMFiles/publications/parq/par-q.pdf.

38. Carter JB, Banister EW, Blaber AP. The effect of age and gender on heart rate variability after endurance training. Med Sci Sports Exerc. 2003:35(8):1333-40.

39. Sharman JE, et al. Augmentation index, left ventricular contractility, and wave reflection. Hypertension. 2009;54(5):1099-105.

40. ACSM. ACSM's resource manual for guidelines for exercise testing and prescription. 8th ed. Philadelphia: Wolters Kluwer Health/Lippincott Williams \& Wilkins; 2010.

41. Nana A, et al. Effects of daily activities on dual-energy X-ray absorptiometry measurements of body composition in active people. Med Sci Sports Exerc. 2012;44(1):180-9.

42. Nana A, et al. Effects of exercise sessions on DXA measurements of body composition in active people. Med Sci Sports Exerc. 2013;45(1):178-85.

43. Melville GW, et al. Fifteen minutes of chair-based yoga postures or guided meditation performed in the office can elicit a relaxation response. Evid Based Complement Alternat Med. 2012;2012:9.

44. Tracy B, Hart C. Bikram yoga training and physical fitness in healthy young adults. J. Strength Cond. Res. 2013;27(3):822-30.

45. Fritz, M.L., et al.. Acute metabolic, cardiovascular, and thermal responses to a single session of Bikram yoga, in Presented at Rocky Mountain ACSM Annual Meeting (Denver, Colorado). 2013.

46. Pate $J$, Buono MJ. The Physiological Responses to Bikram Yoga in Novice and Experienced Practitioners. Altern Ther Health Med. 2014:20(4):12-8.

47. Smith JA, et al. Is there more to yoga than exercise? Altern Ther Health Med. 2011;17(3):22-9.

48. Abel A, et al. Physiological characteristics of long-term Bikram yoga practictioners. J Exerc Physiol Online. 2012:15(5):32-9.

49. Pal $\mathrm{A}$, et al. Effect of yogic practices on lipid profile and body fat composition in patients of coronary artery disease. Complement. Ther. Med. 2011:19(3):122-7.

50. Nagarathna R, et al. Efficacy of yoga based life style modification program on medication score and lipid profile in type 2 diabetes-a randomized control study. Int J Diabetes Dev Countries. 2012;32(3):122-30. 
51. Cade WT, et al. Yoga lifestyle intervention reduces blood pressure in HIV-infected adults with cardiovascular disease risk factors. HIV Med. 2010;11(6):379-88.

52. Brunt $\mathrm{VE}$, et al. Passive heat therapy improves endothelial function, arterial stiffness, and blood pressure in sedentary humans. J Physiol. 2016;594:532942. doi:10.1113/JP272453.

53. Imamura $\mathrm{M}$, et al. Repeated thermal therapy improves impaired vascular endothelial function in patients with coronary risk factors. J Am Coll Cardiol. 2001;38(4):1083-8.

54. Sharma M, Knowlden AP. Role of yoga in preventing and controlling type 2 diabetes mellitus. J Evid. Based Complement. Alternat. Med. 2012;17(2):88-95.

55. Shete SU, Kulkarni DD, Thakur G. Effect of yoga practices on Hs-CRP in Indian railway engine drivers of metropolis. Recent Res Sci Technol. 2012; 4(2):30-3.

56. Pullen PR, et al. Benefits of yoga for African American heart failure patients. Med Sci Sports Exerc. 2010;42(4):651-7.

57. Clay CC, et al. The metabolic cost of hatha yoga. J. Strength Cond. Res. 2005;19(3):604-10.

58. Kristal AR, et al. Yoga practice is associated with attenuated weight gain in healthy, middle-aged men and women. Altern Ther Health Med. 2005;11(4):28-33.

59. Moher D, Schulz KF, Altman DG. The CONSORT statement: revised recommendations for improving the quality of reports of parallel-group randomised trials. Lancet. 2001;357(9263):1191-4.

Submit your next manuscript to BioMed Central and we will help you at every step:

- We accept pre-submission inquiries

- Our selector tool helps you to find the most relevant journal

- We provide round the clock customer support

- Convenient online submission

- Thorough peer review

- Inclusion in PubMed and all major indexing services

- Maximum visibility for your research

Submit your manuscript at www.biomedcentral.com/submit
Biomed Central 